HNO 2021 · 69:705-711

https://doi.org/10.1007/s00106-021-01053-w

Angenommen: 3. Februar 2021

Online publiziert: 31. Mai 2021

๑ Der/die Autor(en) 2021

Berit Schneider-Stickler

Klinische Abteilung Phoniatrie-Logopädie, Univ.-HNO-Klinik, Medizinische Universität Wien, Wien, Österreich

\title{
Stimmdiagnostik bei phonochirurgischen Eingriffen
}

werden Eingriffe an den Stimmlippen bis heute oft noch ausschließlich aufgrund morphologischer Befundkonstellationen ohne Einbeziehung stimmleistungsbeurteilender Kriterien indiziert. ReinkeÖdeme, oft Zufallsbefunde im Rahmen einer Hals-Nasen-Ohren-ärztlichen Untersuchung, werden noch immer eher zu phonochirurgischen Interventionen eingeteilt als winzige Stimmlippenrandverdickungen bei professionellen Stimmnutzern. Während ein Reinke-Ödem nicht selten bei Betroffenen kein Stimmstörungsgefühl verursacht, können feine Stimmlippenrandverdickungen, die oft nur mit hochqualitativen laryngostroboskopischen Untersuchungstechniken visualisiert werden können, bei professionellen Stimmnutzern existenzbedrohende berufliche Einschränkungen verursachen.

Phonochirurgie kann nur dann verantwortungsbewusst praktiziert werden, wenn die ausführenden Chirurg*innen die Eingriffe unter Bewertung der jeweiligen Funktionseinschränkung der Stimme detailliert beurteilen und planen. Die professionelle Analyse der menschlichen Stimme vor und nach phonochirurgischen Eingriffen sollte dabei genauso bewusst und differenziert erfolgen wie die audiologische Diagnostik vor und nach ohrchirurgischen Eingriffen. Die Planung phonochirurgischer Techniken basiert nicht nur auf exakten feinmotorischen Fähigkeiten und der Kenntnis möglicher phonochirurgischer Finessen, sondern auch auf der Kenntnis der möglichen Einflussnahme auf eines der wichtigsten menschlichen Ausdrucksund Arbeitsmittel: der Stimme.

Für die dafür notwendigen präoperativen Untersuchungen stehen mittler- weile eine Vielzahl hochwertiger Untersuchungsmöglichkeiten und internationale Empfehlungen für standardisierte stimmdiagnostische Vorgehensweisen zur Verfügung.

\section{》) Im Klinikalltag sollte ein kompakter stimmdiagnostischer Untersuchungsablauf angestrebt werden}

In Anbetracht der stetig steigenden Anzahl stimmdiagnostischer Möglichkeiten, insbesondere in der wissenschaftlichen Literatur, sollte im Klinikalltag eine kompakte, praktikable und qualitätssichernde Variante eines stimmdiagnostischen Untersuchungsablaufs angestrebt werden.

Behandlungsbedarf, Behandlungszeitpunkt und Behandlungsplan ergeben sich immer aus den individuellen stimmlichen Ansprüchen der Patient*innen.

Die Entscheidung zur Phonochirurgie sollte dann getroffen werden, wenn die jeweilige Befundkonstellation eine Verbesserung bzw. Normalisierung des nachweislichen stimmlichen Defizits erwarten lässt. Die phonochirurgischen Interventionsmöglichkeiten sind je nach zugrunde liegender Pathologie vielfältig $[1,2]$. Perioperative stimmdiagnostische Untersuchungen ermöglichen gezielte quantitative Vergleiche des Therapieerfolgs vor und nach phonochirurgischen Interventionen. Routinemäßige prä- und postoperative stimmdiagnostische Untersuchungen können nicht nur zur Operationsplanung und zum Nachweis von Operationserfolgen, sondern auch zum Vergleich verschiedener phonochirurgi- 


\section{Leitthema}

Tab. 1 Empfohlene multimodale Stimmdiagnostik für die funktionelle Stimmbeurteilung basierend auf dem Basisprotokoll der Europäischen Laryngologischen Gesellschaft. (Mod. in [6])

\begin{tabular}{|c|c|c|}
\hline Komponente & Beispiele & Bewertung \\
\hline Perzeption & $\begin{array}{l}\text { Stimmklangbeurteilung nach der } \\
\text { GRBAS-Skala oder RBH-Skala }\end{array}$ & $\begin{array}{l}\text { 4-Punkte-Skala: } \\
0=\text { keine Abweichung, } 1 \text { = geringgradige } \\
\text { Störung, } 2=\text { mittelgradige Störung, } \\
3=\text { hochgradige Störung }\end{array}$ \\
\hline \multirow{6}{*}{$\begin{array}{l}\text { Visuelle Be- } \\
\text { urteilung der } \\
\text { Stimmlippen- } \\
\text { schwingung } \\
\text { (Laryngostrobo- } \\
\text { skopie) }\end{array}$} & $\begin{array}{l}\text { Beurteilung der Stimmlippen- } \\
\text { schwingungen: }\end{array}$ & \multirow[t]{6}{*}{ Beschreibend } \\
\hline & Glottisschluss & \\
\hline & Regularität/Irregularität & \\
\hline & Schwingungsamplituden & \\
\hline & Randkantenverschieblichkeit & \\
\hline & Symmetrie & \\
\hline \multirow{6}{*}{$\begin{array}{l}\text { Akustische Mes- } \\
\text { sungen }\end{array}$} & $\begin{array}{l}\text { Periodizitätsanalysen } \\
\text { (Jitter = Frequenzvariationen, } \\
\text { Simmer = Amplituden- } \\
\text { variationen) }\end{array}$ & Angabe in \% \\
\hline & Harmonics-to-Noise-Ratio ${ }^{a}$ & Angaben in $\mathrm{dB}$ \\
\hline & Stimmfeldmessungen, z. B.: & \\
\hline & Höchste Frequenz & Angabe in $\mathrm{Hz}$ \\
\hline & Geringste Intensität & Angabe in $\mathrm{dB}$ \\
\hline & Tonhöhenumfang & Angabe in Halbtonschritten \\
\hline \multirow{3}{*}{$\begin{array}{l}\text { Aerodynamische } \\
\text { Untersuchungen }\end{array}$} & Maximale Tonhaltedauer/a:/ & Angabe in $s$ \\
\hline & $\begin{array}{l}\text { Phonationsquotient (Vitalka- } \\
\text { pazität/maximale Tonhalte- } \\
\text { dauer) }\end{array}$ & Angabe in $\mathrm{ml} / \mathrm{s}$ \\
\hline & Vitalkapazität & Angabe in $\mathrm{ml}$ \\
\hline $\begin{array}{l}\text { Subjektive Be- } \\
\text { wertung durch } \\
\text { den Patienten }\end{array}$ & $\begin{array}{l}\text { Beurteilung der Stimmqualität } \\
\text { mithilfe stimmbezogener Fragen }\end{array}$ & Beschreibend/Scores \\
\hline \multicolumn{3}{|c|}{$\begin{array}{l}\text { GRBAS "Grade, roughness, breathiness, asthenic, strained qualities", d. h. Grad der Heiserkeit, Rauigkeit, } \\
\text { Behauchtheit, Asthenie/Schwachheit, Gepresstheit/Angestrengtheit; RBH Rauigkeit, Behauchtheit, } \\
\text { Heiserkeit } \\
\text { aHarmonic-to-Noise-Ratio = Verhältnis zwischen harmonischen und nichtharmonischen Geräuschan- } \\
\text { teilen des Stimmklangs }\end{array}$} \\
\hline
\end{tabular}

scher Vorgehensweisen und alternativer Behandlungstechniken herangezogen werden. Mit zunehmendem Anspruch der Patient*innen an ärztliche Leistungen müssen auch mögliche forensische Aspekte berücksichtigt werden. Stimmdiagnostische Untersuchungen können dabei einerseits zur Argumentation bei unrealistischen Therapiewünschen und andererseits bei eventueller patientenbezogener Unzufriedenheit zur Beweisführung des tatsächlichen Therapieerfolgs nach Phonochirurgie herangezogen werden.

\section{Anamneseerhebung}

Die Anamnese gilt allgemein als Schlüssel zur Diagnostik von Krankheiten. Die Anamneseerhebung in der laryngo- medikationen oder Operationen in der Vergangenheit in Zusammenhang.

Im Rahmen des Anamnesegesprächs müssen bereits absolvierte Therapien ebenso wie stimmtherapeutische Erwartungen erfragt werden.

\section{Standardisierte Stimm- diagnostik}

Im Folgenden wird ein Überblick über die Systematik einer standardisierten Stimmdiagnostik gegeben. International kommen derzeit in der Stimmdiagnostik eine Vielzahl subjektiver, semiobjektiver und phonochirurgischer Untersuchungsmethoden zur Anwendung, die zum großen Teil auf individuellen Beurteilungskriterien beruhen und von regionalen bzw. überregionalen Einflüssen geprägt sind. Für die Diagnosestellung und Bewertung von Therapieergebnissen sind systematische und valide multimodale Untersuchungskonzepte insbesondere im Hinblick auf phonochirurgische Therapieplanungen nach einem standardisierten Protokoll notwendig.

Europäische Phoniater und Laryngologen bemühen sich seit Jahrzehnten, mit multiparametrischen Konzepten der unüberschaubaren Vielfalt an stimmdiagnostischen Untersuchungsmethoden entgegenzuwirken [3-5]. Es ist eine Aufgabe der Zukunft, diese in der täglichen Arbeit umzusetzen.

logischen/phoniatrischen Ambulanz ist i. d. R. eine offene Befragung und schließt sämtliche Fragen zur Erhebung der jetzigen Anamnese, der früheren Anamnese, der Familienanamnese und der sozialen Anamnese ein. Dabei stehen Fragen zu möglichen auslösenden Ereignissen/Art/ Dauer/Qualität der Stimmbeschwerden im Vordergrund. Es gilt abzuklären, ob die Stimmstörungen im Zusammenhang mit beruflicher und/oder privater Stimmbeanspruchung auftreten und ob sie diese beeinträchtigen. Stimmliche Risikofaktoren (Umgebungslärm, Rauchen, Alkohol, Begleiterkrankungen) sind gezielt $\mathrm{zu}$ erfragen. Immer wieder bringen Patient ${ }^{*}$ innen bestehende Stimmprobleme nicht mit früheren Ereignissen, Vorerkrankungen, Dauer-

\section{》) Durch die Europäische Laryngologische Gesellschaft wurde ein stimmdiagnostischer Bewertungsstandard festgelegt}

Nach Eysholdt (2014) stellt die Phonochirurgie als Elektiveingriff besondere Anforderungen an eine korrekte Indikationsstellung mit entsprechender Befunddokumentation [5]. Standardmäßig sollten (Laryngo-)Stroboskopie, Aerodynamikmessungen sowie Stimmaufnahmen und -analysen aus einem gehaltenen Ton kombiniert mit psychometrischen Fragebögen zur Selbst- und Fremdbewertung erfolgen. Durch die Europäische Laryngologische Gesellschaft 
Hier steht eine Anzeige.

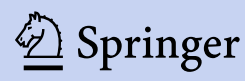


(ELS) per Konsens festgelegt, sind sie seit 2001 der Bewertungsstandard, anhand dessen Indikationsstellung und Behandlungserfolg bewertet werden können. Die ELS definiert als Basisstandard folgende stimmdiagnostische Säulen [4]:

- perzeptive (Fremd-)Beurteilung des

Stimmklangs (Perzeption),

- visuelle Beurteilung der Stimmlip-

penschwingung (Laryngostroboskopie),

- apparative Stimmanalyse und Stimmleistungsbeurteilung (Akustik),

- Erfassung ausgewählter aerodynamischer Parameter (Aerodynamik),

- standardisierte Selbsteinschätzung der eigenen Stimme durch den Patienten (Selbstbeurteilung).

In $\bullet$ Tab. 1 sind die Empfehlungen für die multimodale Stimmdiagnostik, basierend auf dem Basisprotokoll der ELS, mit Beispielen und Bewertungen modifiziert zusammengefasst.

Eine standardisierte Stimmdiagnostik verfolgt das Ziel der Objektivierung und Dokumentation stimmtherapeutischer Entscheidungen und dient der Qualitätssicherung in der Medizin.

Im klinischen Alltag wird die Erhebung stimmdiagnostischer Befunde i. d. R. durch die professionelle Zusammenarbeit zwischen Laryngolog*innen/ Phoniater*innen und Logopäd*innen ermöglicht. Praktisches Beispiel für ein standardisiertes interdisziplinäres Vorgehen ist das Zürcher Stimmdiagnostikprotokoll [7].

Regelmäßiger Erfahrungsaustausch, interne Supervision und wissenschaftliche Fortbildungen sichern die einrichtungsinterne Qualität der stimmdiagnostischen Befunderhebung und -interpretation.

\section{Perzeption}

Leitsymptom einer Stimmstörung ist die Heiserkeit, die die auditiv wahrnehmbaren Abweichungen vom normalen Stimmklang zusammenfasst. Bei jedem Patient ${ }^{*}$ innengespräch findet, ob bewusst oder unbewusst, eine „psychoakustische“ Beurteilung des Stimmklangs über das Ohr de ${ }^{\star} r$ Untersucher ${ }^{*}$ in im
Sinne einer auditiv-perzeptiven Stimmklangbeurteilung statt.

International haben sich 2 Bewertungssysteme durchgesetzt, zum einen die GRBAS-Skala („grade, roughness, breathiness, asthenic, strained qualities") nach Hirano und zum anderen die davon gekürzte RBH-Skala (Rauigkeit, Behauchtheit, Heiserkeit) nach Wendler et al. $[8,9]$. Beide definieren die Heiserkeit als auditives Leitsymptom einer Stimmstörung, deren Teilkomponenten zusätzlich beschrieben werden sollen (•Tab. 2).

Die Parameter werden entsprechend ihrer Ausprägung auf einer 4-PunkteSkala graduiert:

- $0=$ nicht vorhanden,

- 1 = geringgradig vorhanden,

$-2=$ mittelgradig vorhanden,

- 3 = hochgradig vorhanden.

Der Gesamtgrad der Heiserkeit (G bzw. H) entspricht in der Gesamtbeurteilung nicht der Summe der anderen Faktoren, sondern der höchsten Beurteilung der Einzelfaktoren.

I) Der Gesamtgrad der Heiserkeit entspricht der höchsten Beurteilung der Einzelfaktoren

Die perzeptiv-auditive Stimmklangbeurteilung der Stimmklangparameter erfolgt i. d. R. für Spontansprache und für Standardtexte.

\section{Laryngostroboskopie}

Erst die Kombination der Laryngoskopie mit einer Stroboskopie ermöglicht die visuelle Beurteilung der phonatorischen Stimmlippenmotilität und damit der Schwingungscharakteristika der Stimmlippen. Laryngostroboskopien sind sowohl starr als auch flexibel inzwischen mit exzellenter Bild- und Lichtqualität möglich. Digitale Befundaufzeichnungen sind inzwischen für Begutachtungen sowie prä- und postoperative Befundvergleiche üblicher Standard. Auch für phonochirurgische Überlegungen sind stroboskopische Auf-
HNO 2021 · 69:705-711

https://doi.org/10.1007/s00106-021-01053-w

(c) Der/die Autor(en) 2021

\section{B. Schneider-Stickler}

\section{Stimmdiagnostik bei phonochirurgischen Eingriffen}

\section{Zusammenfassung}

Stimmdiagnostische Untersuchungen vor und nach phonochirurgischen Eingriffen dienen zum einen der detaillierten Planung der chirurgischen Vorgehensweise und zum anderen der Nachvollziehbarkeit des postoperativen Ergebnisses. Die Stimmdiagnostik verfolgt dabei eine vielschichtige Vorgehensweise und umfasst Aspekte aus perzeptiver Stimmklangbeurteilung, Laryngostroboskopie, Stimmakustik, Aerodynamik und der Selbsteinschätzung durch die Patient*innen. Die Auswahl stimmdiagnostischer Methoden sollte sich dabei immer am aktuellen Wissensstand orientieren und das einrichtungsinterne Vorgehen prägen.

\section{Schlüsselwörter}

Diagnostische Techniken und Verfahren . Diagnostische Selbstbeurteilung - Endoskopie -Stimmqualität $\cdot$ Symptombeurteilung . Phonochirurgie

\section{Voice diagnostics in phonosurgical interventions}

\section{Abstract}

Voice diagnostics before and after phonosurgical interventions should aim at detailed planning of surgical techniques and evaluability of postoperative outcome. They should consider multimodal concepts and include measurements of perceptual voice analysis, laryngostroboscopy, voice acoustics, aerodynamics, and self-evaluation by the patients. Selected methods for voice diagnostics should reflect current knowledge and define the departmental procedure.

Keywords

Diagnostic techniques and procedures . Diagnostic self-evaluation - Endoscopy . Voice quality - Symptom assessment . Phonosurgery 


\begin{tabular}{|c|c|c|c|}
\hline Abk. & $\begin{array}{l}\text { Englische } \\
\text { Bezeich- } \\
\text { nung }\end{array}$ & $\begin{array}{l}\text { Deutsche Be- } \\
\text { zeichnung }\end{array}$ & Beschreibung \\
\hline $\mathrm{G}=\mathrm{H}$ & "Grade" & $\begin{array}{l}\text { Gesamtgrad der } \\
\text { Heiserkeit }\end{array}$ & Gesamteindruck der Stimmstörung bzw. der Heiserkeit \\
\hline $\mathrm{R}$ & „Roughness" & Rauigkeit & $\begin{array}{l}\text { Störung des Stimmklangs durch den Eindruck irregulärer } \\
\text { Schwingungsanteile, tieffrequenter Geräuschanteile oder } \\
\text { Vocal-Fry }\end{array}$ \\
\hline B & "Breathiness" & Behauchtheit & $\begin{array}{l}\text { Störung des Stimmklangs durch hörbare turbulente Luft- } \\
\text { strömungsanteile, meist bedingt durch inkompletten } \\
\text { Glottisschluss }\end{array}$ \\
\hline A & "Asthenic" & Schwachheit & $\begin{array}{l}\text { Auditiver Eindruck einer Stimmschwäche wie bei Hypo- } \\
\text { funktion }\end{array}$ \\
\hline$S$ & $\begin{array}{l}\text { "Strained } \\
\text { qualities" }\end{array}$ & $\begin{array}{l}\text { Gepresstheit/ } \\
\text { Angestrengt- } \\
\text { heit }\end{array}$ & $\begin{array}{l}\text { Auditiver Eindruck eines übermäßigen Spannungs- und } \\
\text { Anstrengungsgrades wie bei einer Hyperfunktion }\end{array}$ \\
\hline
\end{tabular}

nahmen zur Beurteilung der Schwingungseigenschaften der Stimmlippen unverzichtbar, da nicht nur der Organbefund selbst, sondern sein Einfluss auf die Stimmlippenschwingungscharakteristik ausschlaggebend für die Wahl phonochirurgischer Techniken ist. Laryngostroboskopische Untersuchungen sollten sowohl für Grundfrequenzen im Bereich der indifferenten Sprechstimmlage als auch bei hoher Phonation mit verschiedenen Intensitäten erfolgen. Die klinische Beurteilung orientiert sich im Wesentlichen an den bekannten stroboskopischen Parametern: Schwingungsamplituden, Randkantenverschieblichkeit, Schwingungssymmetrie, Irregularitäten, Phasendifferenzen und Glottisschluss [6, 10].

Die digitale Aufzeichnung der Untersuchungsbefunde ermöglicht eine spätere Befundeinsicht für Patientenbesprechungen, prä- und postoperative Vergleiche und spätere Befundauswertungen.

Seit einigen Jahren werden in einigen Stimmzentren Endoskopien mit hoher Zeitauflösung verwendet, die Hochgeschwindigkeitsendoskopie $[5,11,12]$. Mit dem Fokus auf zusätzlichen Bewegungsinformationsgewinn werden Bilddaten in Form von „Phonovibrogrammen" komprimiert, die die beidseitigen Stimmlippenbewegungen im Seitenvergleich im Zeitverlauf objektivieren.

\section{Akustik}

Aus einem Stimmsignal lassen sich zahlreiche Maße zur Beschreibung einer Stimme ermitteln.

Die akustische Analyse von Stimmklängen gehört in der standardisierten Stimmdiagnostik zu den unverzichtbaren Untersuchungsverfahren zur Objektivierung und Quantifizierung der akustischen Eigenschaften der Stimme. Voraussetzung dafür ist die Aufnahme und Speicherung repräsentativer Stimmsignale (Standardtexte, Standardsätze, ausgehaltene Vokale) unter standardisierten Aufnahmebedingungen (u.a. definierter Mund-Mikrofon-Abstand, Mikrofonauswahl, geräuscharme Umgebung).

In der Literatur existiert mittlerweile eine unendliche Vielzahl akustischer Stimmparameter, die jedoch oft wissenschaftlichen Fragestellungen vorbehalten sind und ein gewisses Maß an physikalisch-akustischer Expertise voraussetzen. Gelegentlich überschreiten sie jedoch das gängige praxisorientierte stimmdiagnostische Fachwissen. Einige wesentliche Parameter basierend auf Perturbationsmessungen bzw. auf spektralanalytischen Verfahren haben dennoch Eingang in die klinische Stimmdiagnostikroutine gefunden und werden bereits in einigen kommerziell erhältlichen Stimmdiagnostik-Softwareprogrammen eingebunden. Dazu zählen in erster Linie die Perturbationsparameter Jitter (mittlere Abweichung der Schwingungsfrequenz) und Shimmer (mittlere Abweichung der
Schwingungsamplitude), als auch die Harmonic-to-Noise-Ratio (Verhältnis zwischen harmonischen und nichtharmonischen Geräuschanteilen des Stimmklangs/Harmonische-GeräuschVerhältnis).

\section{》) Die Stimmfeldmessung gilt als „Arbeitspferd“ zur Beurteilung der stimmlichen Leistung}

Für die Beurteilung der Stimmleistung steht die Stimmfeldmessung (von einigen Autor*innen auch Stimmprofilmessung genannt) zur Verfügung. Die Stimmfeldmessung gilt als "Arbeitspferd“ zur Beurteilung der stimmlichen Leistung bzw. stimmlicher Defizite und ist in der klinischen Bedeutung vergleichbar mit der Tonaudiometrie in der Otologie. In einem $x$ - $y$-Diagramm ( $x=$ Frequenz, $\mathrm{y}=$ Schalldruckpegel) werden Sprechstimmleistung (in verschiedenen Steigerungsstufen von leiser Stimmgebung bis zur Rufstimme) und Singstimmleistung (Tonhöhenumfang bei leisem und lautem Singen) dokumentiert. Die Stimmfeldmessung ist eine Momentaufnahme der stimmlichen Leistung zum Untersuchungszeitpunkt, jedoch gibt sie Aufschluss über eine Vielzahl stimmlicher Beurteilungskriterien hinsichtlich Stimmkonstitution und Stimmfunktion.

Besondere Aufschlüsse bieten hierbei minimal und maximal erreichbare Intensitäten der Sing- und Sprechstimme und der Tonhöhenumfang der Singstimme. Die minimale Intensität soll dabei mit dem Ansprechen der Stimmlippenschwingungen korrelieren und Ausdruck der Geschmeidigkeit des Stimmlippenepithels und der Grundspannung der Stimmlippe sein. Fixierungen oder Versteifungen im Stimmlippenepithel bzw. im Subepithelialraum lassen Stimmlippenschwingungen erst bei höheren Intensitäten zu. Darüber hinaus lassen sich aus den Messergebnissen stimmkonstitutionelle und -konditionelle Rückschlüsse ziehen.

Derzeit inkludieren verschiedene kommerzielle Softwareprogramme die multiparametrische Auswertung der Stimmqualität mithilfe des DysphoniaSeverity-Index (DSI) nach Wuyts et al. 
[13]. Der DSI soll über den Grad der Dysphonie und die Stimmqualität unter Verwendung von 4 Parametern informieren:

- höchste im Stimmfeld erreichte

Frequenz (F0-high in $\mathrm{Hz}$ ),

- geringster im Stimmfeld erreichter Schalldruckpegel/Intensität (I-low in dB),

- maximale Tonhaltedauer/

Phonationszeit auf /a:/ (MPT

in s),

- Jitter (in \%).

\section{Aerodynamik}

Die einfachste und am leichteste durchzuführende stimmdiagnostische Messung ist die Messung der maximalen Tonhaltedauer eines mit mittlerer Intensität und auf Sprechtonhöhe ausgehaltenen Vokals /a:/.

Im Idealfall ist die zusätzliche spirometrische Bestimmung der Vitalkapazität möglich, die man zur Berechnung des Phonationsquotienten (PQ, Verhältnis aus Vitalkapazität und maximaler Tonhaltedauer) verwenden kann.

Die Berücksichtigung der Vitalkapazität im Rahmen der Stimmdiagnostik kann auf zusätzliche Lungenfunktionsprobleme hinweisen, die die Stimmleistung beeinträchtigen können, ohne dass primär ein Stimmproblem zugrunde liegt.

\section{Subjektive Einschätzung durch Patient*innen}

Phonochirurgische Interventionen sind im Gegensatz zu beispielsweise onkologischen Operationen keine vital notwendigen Eingriffe. In der phonochirurgischen Therapieplanung haben daher die Wünsche und stimmlichen Bedürfnisse einen besonderen Stellenwert [3]. Jede phonochirurgische Intervention muss sich dem Therapiewunsch der Patient ${ }^{*}$ innen unterordnen. Daher hat die präoperative Selbsteinschätzung der stimmlichen Situation und des möglichen stimmlichen Handicaps einen besonderen Stellenwert, um in der Zusammenschau von stimmdiagnostischen Befunden, phonochirurgischen Interventionsmöglichkeiten und individuellen Patient ${ }^{*}$ innenansprüchen ein gemeinsames Therapiekonzept von Operateur*innen und Patient*innen zu erreichen.

\section{》) Die systematisierte \\ Selbstauskunft mithilfe von standardisierten Fragebögen ist essenziell}

Fragebögen können zur Reflexion der gefühlten Stimmstörung und zur Beurteilung der Teilhabe am gesellschaftlichen Kommunikationsprozess verstanden werden und sollten erhobene klinische Untersuchungsbefunde ergänzen. Die systematisierte Selbstauskunft von Patient*innen mithilfe von standardisierten Fragebögen ist für die Diagnostik von Stimmstörungen und deren Therapie essenziell.

In Anbetracht einer inzwischen unendlichen Vielfalt stimmdiagnostischer Möglichkeiten ist es im Klinikalltag empfehlenswert, sich auf eine kompakte qualitätssichernde Minimalvariante eines stimmdiagnostischen Untersuchungsablaufs zu konzentrieren.

Nicht selten gehen minimale funktionelle oder organische Auffälligkeiten mit einem hohen Leidensdruck einher. Andere Patient*innen haben trotz ausgeprägter organischer Befunde nur geringe subjektive stimmliche Einbußen. Für die Planung phonochirurgischer Interventionen ist der Behandlungswunsch der Patient ${ }^{*}$ innen anhand der stimmlichen Selbsteinschätzung maßgeblich.

Die Abfrage der subjektiven Erfahrung der Betroffenen anhand von Selbsteinschätzungsbögen spielt darüber hinaus bei vergleichenden Wirksamkeitsstudien eine große Rolle [14, 15]. Nach einem systematischen Review von Salm et al. stehen im Deutschen verschiedene Fragebögen zur Auswahl [16]: - Classical and Modern Singing Handicap Index (CSHI/MSHI) [17],

- Stimmstörungsindex (SSI = VHI-12) [18],

- Singing Voice Handicap Index (SVHI) [19],

- Transsexual Voice Questionnaire for Male-to-Female Transsexuals (TVQMtF) [20],
- Voice Handicap Index (VHI-30) [21],

- VHI-9 [22],

- Voice-related Quality of Life Questionnaire (V-RQOL) [23, 24].

Von diesen Fragebögen findet in der klinischen Praxis in erster Linie der Voice Handicap Index in seiner ursprünglichen Version mit 30 Items breite Anwendung. Dieser dient der Selbsteinschätzung bei Heiserkeit und/oder einem gestörten Stimmklang durch Beantwortung von 30 Fragen auf einer Bewertungsskala von 0 (nie), 1 (selten), 2 (manchmal), 3 (oft) bis 4 (immer). Kritik an diesem Fragebogen wurde immer wieder aufgrund des hohen Zeitaufwands für das Ausfüllen des Fragebogens geübt, weshalb er inzwischen in gekürzten Fassungen als SSI = VHI-12 mit 12 Fragen bzw. VHI-9 mit 9 Fragen zur Anwendung kommt. Für die gezielte Befragung von Sänger*innen mit Stimmproblemen hat sich der mehr auf die Sprechstimme fokussierte VHI30 (einschließlich VHI-9 und VHI-12) nicht bewährt, sodass für die Erfassung der gesanglichen Einschränkungen der Sänger*innen speziell der SVHI als diagnostisches Selbstbeurteilungsinstrument entwickelt wurde. Wie beim VHI-30 ist die Beantwortung der Fragen auf einer Bewertungsskala mit 0 (keine Störung), 1 (leicht gestört), 2 (mittelgradig gestört) bis 3 (hochgradig gestört möglich). Zur Beurteilung des Einflusses einer Stimmstörung auf die Lebensqualität bzw. der stimmbezogenen Lebensqualität kann der V-RQOL eingesetzt werden. Dieser besteht aus 10 Items, davon sind 4 Items sozial-emotionalen Aspekten und 6 Items physisch-funktionellen Aspekten zuzuordnen. Die Antwortkategorien reichen von 1 (kein Problem), 2 (kaum ein Problem), 3 (schon ein Problem), 4 (ein großes Problem) bis 5 (ein Problem, wie es schlimmer nicht sein könnte).

Mit den Fragebögen „Classical und Modern Singing Handicap Index “ (CSHI/MSHI) soll gezielt der Einfluss stimmlicher Beeinträchtigungen auf die Lebensqualität von Sänger*innen erhoben werden. Mit den unterschiedlichen Versionen für einerseits klassische Sänger*innen (CSHI) und andererseits nichtklassische Sänger*innen (MSHI) sollen die unterschiedlichen Anforde- 
rungen des jeweiligen Gesangsstils Berücksichtigung finden. Beide Fragebögen bestehen aus 30 Items. Jeweils 10 Items überprüfen die Auswirkungen der Probleme auf die professionelle Sing- und Sprechstimme („disability"), psychologische Auswirkungen („handicap“) und die Wahrnehmung der Qualitätsmerkmale der Singstimme („impairment"). Die Selbsteinschätzung erfolgt in der deutschen Version auf einer 4-stufigen Skala mit 0 (nie), 1 (manchmal), 2 (oft) und 3 (immer).

Der TVQMtF ist speziell der Selbsteinschätzung von Mann-zu-Frau-Transsexuellen (Trans ${ }^{\star}$ Frauen) vorbehalten. In 30 Items werden verschiedene erlebte Situationen der Stimmanwendung und -akzeptanz in verschiedenen Lebenssituationen hinterfragt. Hier sind die Beantwortungen auf einer 4-stufigen LikertSkala mit den Stufen 1 (nie oder selten), 2 (manchmal), 3 (oft) und 4 (meist oder immer) möglich. Die Auswertung fokussiert dabei auf die Einschätzung, wie weiblich bzw. männlich die eigene Stimme wahrgenommen wird und wie weiblich bzw. männlich sie idealerweise klingen sollte.

\section{Fazit für die Praxis}

- Stimmdiagnostische Untersuchungen vor und nach phonochirurgischen Eingriffen sind unerlässlich.

- Sie unterstützen zum einen die detaillierte Operationsplanung und dienen zum anderen der Qualitätssicherung in der Medizin.

- Fundierte stimmphysiologische und stimmakustische Kenntnisse sind Voraussetzung für eine evidenzbasierte Stimmdiagnostik.

- Die standardisierte Stimmdiagnostik verfolgt dabei eine vielschichtige Vorgehensweise und umfasst Aspekte aus perzeptiver Stimmklangbeurteilung, Laryngostroboskopie, Stimmakustik, Aerodynamik zur Stimmfunktion und Selbsteinschätzung durch Patient*innen.

- Die Auswahl stimmdiagnostischer Methoden sollte sich dabei immer am aktuellen Wissensstand orientieren und das einrichtungsinterne Vorgehen prägen.

\section{Korrespondenzadresse}

Ao. Univ. Prof. Dr. med. Berit SchneiderStickler

Klinische Abteilung Phoniatrie-Logopädie, Univ.-HNO-Klinik, Medizinische Universität Wien

Währinger Gürtel 18-20, 1090 Wien, Österreich berit.schneider-stickler@meduniwien.ac.at

Funding. Open access funding provided by Medical University of Vienna.

\section{Einhaltung ethischer Richtlinien}

Interessenkonflikt. B. Schneider-Stickler gibt an, dass kein Interessenkonflikt besteht.

Für diesen Beitrag wurden von den Autoren keine Studien an Menschen oder Tieren durchgeführt. Für die aufgeführten Studien gelten die jeweils dort angegebenen ethischen Richtlinien.

Open Access. Dieser Artikel wird unter der Creative Commons Namensnennung 4.0 International Lizenz veröffentlicht, welche die Nutzung, Vervielfältigung, Bearbeitung, Verbreitung und Wiedergabe in jeglichem Medium und Format erlaubt, sofern Sie den/die ursprünglichen Autor(en) und die Quelle ordnungsgemäß nennen, einen Link zur Creative Commons Lizenz beifügen und angeben, ob Änderungen vorgenommen wurden.

Die in diesem Artikel enthaltenen Bilder und sonstiges Drittmaterial unterliegen ebenfalls der genannten Creative Commons Lizenz, sofern sich aus der Abbildungslegende nichts anderes ergibt. Sofern das betreffende Material nicht unter der genannten Creative Commons Lizenz steht und die betreffende Handlung nicht nach gesetzlichen Vorschriften erlaubt ist, ist für die oben aufgeführten Weiterverwendungen des $\mathrm{Ma}$ terials die Einwilligung des jeweiligen Rechteinhabers einzuholen.

Weitere Details zur Lizenz entnehmen Sie bitte der Lizenzinformation auf http://creativecommons.org/ licenses/by/4.0/deed.de.

\section{Literatur}

1. Friedrich G, de Jong Fl, Mahieu HF, Benninger MS, Isshiki N (2001) Laryngeal framework surgery: a proposal for classification and nomenclature by the Phonosurgery Committee of the European Laryngological Society. Eur Arch Otorhinolaryngol 258(8):389-396

2. emacle $M$, Friedrich $G$, Dikkers FG, de Jong $F$ (2003) Phonosurgery of the vocal folds: a classification proposal. Eur Arch Otorhinolaryngol 260:1-6

3. Friedrich $G$ (1995) Basic principles for indications in phonosurgery. Laryngorhinootologie 74:663-665

4. Dejonckere PH, Bradley P, Clemente P, Cornut G, Crevier-Buchman L, Friedrich $G$, van de Heyning $P$ Remarcle M, Woissard V (2001) A basic protocol for functional assessment of voice pathology, especially for evaluation of efficacy of (phonosurgical) treatments and evaluating new assessment techniques. Eur Arch Otorhinolaryngol 258:77-82
5. Eysholdt U (2014) Heiserkeit-Biomechanik und quantitative Laryngoskopie. HNO 62(7):541-552

6. Schneider-Stickler B, Bigenzahn W (2013) Stimmdiagnostik-Ein Leitfaden für die Praxis, 2. Aufl. Springer, Wien. ISBN 978-3-7091-1480-3

7. Balandat B, Riedmüller S, Bohlender J (2013) Aus der Praxis für die Praxis:Zürcher Stimmdiagnostik. Forum Logop 4(23):12-17

8. Hirano M (1981) GRBAS' scale for evaluating the hoarse voice \& frequency range of phonation. In: Clinical examination of voice. Springer, Wien New York

9. Wendler J, Rauhut A, Krüger H (1986) Classification of voice qualities. J Phonet 14:483-488

10. Schönhärl E (1960) Die Stroboskopie in der praktischen Laryngologie. Thieme, Stuttgart

11. Lohrscheller J, Eysholdt U (2008) Phonovibrogram visualization of entire vocal fold dynamics. Laryngoscope 118:753-758

12. Lohrscheller J, Eysholdt U, Toy $\mathrm{H}$ et al (2008) Phonovibrography: mapping high-speed movies of vocal fold vibrations into 2-D diagrams for visualizing and analyzing the underlying laryngeal dynamics. IEEE Trans Med Imaging 27:300-309

13. Wuyts FL, De Bodt MS, Molenberghs G, Remarcle M, Heylen L, Millet B, Van Lierde K, Raes J, Van de Heyninhg PH (2004) The dysphonia severity index: an objective measure of vocal quality based on a multiparametric approach. J Speech Lang Hear Res 43:796-809

14. Fayers PM, Machin D (2016) Quality of life: the assessment, analysis and reporting of patientreported outcomes, 3. Aufl. Wiley-Blackwell, West Sussex

15. Koller M, Neugebauer EAM, Augustin M, Büssing A, Farin E, Klinkhammer-Schalke M, Wieseler B (2009) Die Erfassung von Lebensqualität in der Versorgungsforschung: konzeptuelle, methodische und strukturelle Voraussetzungen. Gesundheitswesen 71(12):864-872

16. Salm S, Hartrampf J, Neumann S (2018) Die Erfassung stimmbezogener Lebensqualität ( $\mathrm{Vr}$ QoL) - ein systematisches Review publizierter Assessments. Forsch Sprache 2:3-31

17. Fussi F (2005) La voce del cantante, 3. Aufl. Omega, Torino

18. Nawka T, Gonnermann U (2004) Stimmstörungsindex (SSI). In: Gross M, Kruse E (Hrsg) Aktuelle phoniatrisch-pädaudiologische Aspekte. Median Verlag, Heidelberg, S375-379

19. Cohen SM, Jacobson BH, Garrett CG, Noordzij JP, Stewart MG, Attia A, Cleveland TF (2007) Creation and validation of the singing voice handicap index. Ann Otol Rhinol Laryngol 116(6):402-406

20. Dacakis G, Davies S, Oates JM, Douglas JM, Johnston JR (2013) Development and preliminary evaluation of the transsexual voice questionnaire for male-to-female transsexuals. J Voice 27(3):312-320

21. Jacobson $B H$, Johnson $A$, Grywalski C, Silbergleit $A$, Jacobson G, Benninger MS, Newman CW (1997) The voice handicap index (VHI): development and validation. Am J Speech Lang Pathol 6(3):66-70

22. Nawka T, Verdonck-de Leeuw IM, de Bodt M, Guimaraes I, Holmberg EB, Rosen CA, Konerding U (2009) Item reduction of the voice handicap index based on the original version and on European translations. Folia Phoniatr Logop 61(1):37-48

23. Hogikyan ND, Sethuraman G (1999) Validation of an instrument to measure voice-related quality of life (V-RQOL). JVoice 13(4):557-569

24. Günther S, Rasch T, Klotz M, Hoppe U, Eysholdt U, Rosanowski F (2005) Bestimmung der subjektiven Beeinträchtigung durch Dysphonien. HNO 53:895-900 